\title{
The Effect of Oxygen Potential on the Surface Self-Diffusion Coefficient of $\alpha$-Fe*
}

\author{
By Kazuyoshi Nii** and Kazuhiro Yoshihara**
}

\begin{abstract}
The surface self-diffusivity of $\alpha$-Fe was measured by the grain boundary grooving method in hydrogen atmospheres with dew points of 213 and $286 \mathrm{~K}$. The groove width measurement was made through all runs with the same region of grain boundary of coarse-grained specimens. The diffusion anneal was carried out at 1073, 1123 and $1163 \mathrm{~K}$.

In dry hydrogen (dew point $=213 \mathrm{~K}$ ) purified through a palladium foil, the surface selfdiffusivity of $\alpha-\mathrm{Fe}$ can be expressed by the following equation:

$$
D_{s}=10 \exp (-228 \mathrm{~kJ} / R T)\left(\mathrm{m}^{2} \cdot \mathrm{s}^{-1}\right) \text {. }
$$

The value of activation energy agrees well with that obtained by Blakely et al.

In moist hydrogen (dew point $=286 \mathrm{~K}$ ), however, the rate of groove development was varied, depending on whether or not the specimen had been equilibrated with the atmosphere. When the specimen, which had been in equilibrium with dry hydrogen, was annealed in moist hydrogen, the rate of groove development was larger than that in dry hydrogen in the early stage, but it decreased with annealing time. On the other hand, if the specimen had been equilibrated with the same atmosphere, the grooving rate was smaller than that in dry hydrogen. In the former case, surface roughening would occur more than in thermal equilibrium owing to the adsorption and solution reactions of oxygen at the surface, which would lead to an enhancement of surface diffusivity. On the contrary, on the surface of the specimen, which is equilibrated with moist hydrogen, the blocking of kink and ad-atom would occur by adsorption of oxygen, which would lower the surface diffusivity.

A marked decrease in surface diffusivity in hydrogen with a dew point of $286 \mathrm{~K}$ was observed at $1073 \mathrm{~K}$ with the specimen equilibrated with the same atmosphere, which could be attributed to an incidental adsorption of impurities during the sample preparation.
\end{abstract}

(Received May 7, 1979)

\section{Introduction}

The surface self-diffusivity of metals is an important step for some reaction such as sintering or particle growth. For instance, the voids remained in the iron sinterings are sharp-edged when sintered in moist hydrogen, while rounded in dry hydrogen ${ }^{(1)}$. This indicates that the surface self-diffusivity and thus the contribution of surface self-diffusivity to sintering process has been changed with the oxygen potential in the atmospheres.

The surface self-diffusivity of metals, on the other side, represents the product of mobility and concentration of mobile defects on surface, and thus the change in surface self-diffusivity

* This paper was originally published in Japanese in J. Japan Inst. Metals, 40 (1976), 26.

** National Research Institute for Metals, Meguroku, Tokyo 153, Japan. should be a reflection of the change in surface structure or other surface properties. Therefore, the change in adsorption state of surface can be deduced from the change in surface selfdiffusivity.

The surface self-diffusion of metals, however, has been less fully understood than the volume diffusion or other short-circuit diffusion, because the experimental difficulties are greater in surface diffusion measurement. The surface of metals would be always contaminated by gas adsorption from atmospheres or by segregation of impurities from metal interiors, and, moreover, the degree of contamination is varied with time during the measurement. Therefore, the long-term experiment is very difficult for surface processes. The further difficulties in measurement of surface diffusivity is that the interpretation of isotope experiment is complexed and hardly possible owing to a simultaneous contribution of volume diffusion and grain boundary diffusion.

1979 Vol. 20 
In 1957, however, the theory of grain boundary grooving by surface diffusion was proposed by Mullins and this has been applied to the measurement of surface diffusivity. Since then, the diffusion data by this technique and others have been accumulated, and the following has been reported on the effect of atmospheres on the surface self-diffusivity; the surface self-diffusivities of silver ${ }^{(2)(3)}$ and copper $^{(4) \sim(7)}$ were increased by adsorption of oxygen $^{(2)(4)}$, sulfur $^{(3)(5)}$ or halogens ${ }^{(6)(7)}$, while that of iron ${ }^{(8)}$ was decreased by adsorption of oxygen. The results of these measurements, however, have been widely scattered, and a general interpretation cannot be obtained on the effect of atmospheres on the surface selfdiffusivity.

In the present experiment, therefore, the surface self-diffusivity of $\alpha$-iron has been measured in various oxygen potentials by grain boundary grooving method as a part of the study to draw a general conclusion on the effect of adsorption on the surface selfdiffusivity.

\section{Experimental}

\section{Specimen preparation}

The re-electrolytic iron (Showa Denko) was melted in induction furnace. The ingot was forged and rolled into the bar of $15 \times 15 \mathrm{~mm}$ square, and then strain-annealed to promote the grain growth. A few pieces of $15 \times 15 \times 3$ $\mathrm{mm}$ were cut from the bar, the average grain size being about $5 \mathrm{~mm}$. The orientation of grains was examined by optical reflection from (100) facets, which had been formed by etching in $\mathrm{HNO}_{3}-\mathrm{H}_{2} \mathrm{O}(1: 2)$. A piece that had the adjacent grains in the best symmetric relation was selected, and the symmetry was further confirmed by annealing and observing the symmetry of the groove developed in dry hydrogen. The Laue diffractometry revealed, however, that the orientation of the selected adjacent grains was not so symmetric and not expressed with the simple Miller's index. Nevertheless, the symmetry of the groove developed was good enough to examine the effect of atmosphere on surface self-diffusion.
The iron used contains nickel and cobalt amounting to $0.02 \%$, but these elements are not surface active and thus assumed to have no effect on the surface diffusivity.

The iron piece finally selected for the present experiment was annealed to stabilize the grain boundary and also for decarburization and deoxygenation in moist hydrogen (saturated with water vapor at room temperature) for $50 \mathrm{~h}$ and subsequently in dry hydrogen for $50 \mathrm{~h}$. This specimen was used throughout all runs in the present experiment, and the groove width measurement was made at the same region of the selected boundary.

The specimen was mechanically polished with emery paper and diamond paste, and then electropolished for $10 \sim 20 \mathrm{~min}$ in $\mathrm{CrO}_{3}-$ $\mathrm{CH}_{3} \mathrm{COOH}-\mathrm{H}_{2} \mathrm{O}$ solution, immediately followed by ultrasonic washing with $\mathrm{CH}_{3} \mathrm{COOH}$ for $1 \mathrm{~min}$ and then with distilled water 10 times. The specimen was further cleaned by ion bombardment in Ar of $4 \mathrm{~Pa}$ with $1 \mathrm{kV}$ acceleration voltage for $5 \mathrm{~min}$. On the surface prepared through the above procedures, small amount of $\mathrm{S}, \mathrm{Cl}$ and $\mathrm{K}$ were detected by Auger electron spectroscopy. When the specimen was washed only with distilled water after electropolishing, $\mathrm{Cr}$ was detected on the surface. The washing with $\mathrm{CH}_{3} \mathrm{COOH}$ is, therefore, effective in removing impurities from the surface, but care should be taken not to etch the grain boundary.

\section{Diffusion anneal}

The diffusion anneal was conducted at 1073 $\mathrm{K}, 1123 \mathrm{~K}$ and $1163 \mathrm{~K}$ in dry hydrogen (dew point $=228 \mathrm{~K} \sim 203 \mathrm{~K}$ ) and in moist hydrogen (dew point $=288 \mathrm{~K}$ ), respectively. The specimen was inserted into the hot zone of the furnace, which had been adjusted at the given temperature and atmosphere. During annealing the specimen was covered with an iron lid to prevent the possible development of groove by evaporation. At determined intervals the specimen was pulled out from the hot zone and cooled in the same atmosphere. The interferogram was taken after each diffusion anneal with an interference microscope (Olympus M 1) with use of a Na-lamp. The annealing time was accumulated up to $200 \mathrm{~h}$.

The diffusion anneal in dry hydrogen was 


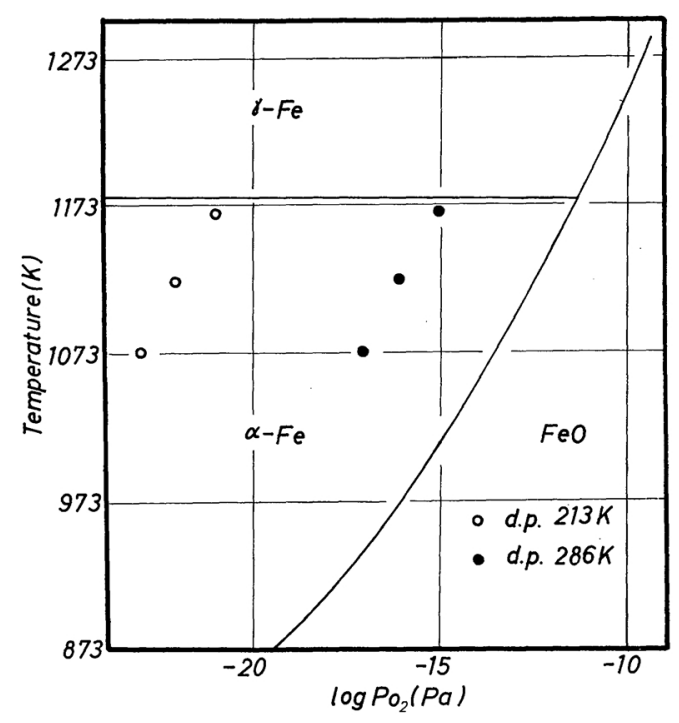

Fig. 1 The experimental conditions.

carried out in the hydrogen which was purified through palladium foil. At the beginning of the run, dew point of hydrogen was $228 \mathrm{~K}$, lowered to $218 \mathrm{~K}$ within $1 \mathrm{~h}$ and sometimes reached $203 \mathrm{~K}$ after $20 \mathrm{~h}$. The dew point on the average was estimated as $213 \mathrm{~K}$. The experiment in moist hydrogen was conducted in the hydrogen which was saturated with water vapor at $286 \mathrm{~K}$ passing through two saturators. The dew point was monitored continuously at the exit of gas flow with a hygrometer (SHAW). The condition of the present experiment are summarized in Fig. 1.

\section{The measurement of groove width and the calculation of surface self-diffusivity}

The groove width was measured on the film by a comparator and averaged for ten lines of the interferogram at the grain boundary selected previously. If the grain boundary grooving occurs through surface diffusion, the groove width is expressed by the following equation ${ }^{(9)}$,

$$
\begin{aligned}
& w=4.6(B t)^{1 / 4}, \\
& B=D_{s} \gamma_{s} \Omega^{2} N / k T,
\end{aligned}
$$

where $w$ : groove width (m)

$t$ : annealing time (s)

$D_{s}:$ surface self-diffusivity $\left(\mathrm{m}^{2} \cdot \mathrm{s}^{-1}\right)$

$\gamma_{s}:$ surface tension $\left(\mathrm{J} \cdot \mathrm{m}^{-2}\right)$

$\Omega:$ atomic volume $\left(\mathrm{m}^{3} \cdot\right.$ atom $\left.^{-1}\right)$
$N$ : surface atomic density $\left(\right.$ atom $\left.\cdot \mathrm{m}^{-2}\right)$

$k$ : Boltzmann's constant $(1.3805 \times$

$$
10^{-23} \mathrm{~J} \cdot \mathrm{K}^{-1} \text { ) }
$$

$T$ : temperature (K)

Although the surface atomic density $N$ depends on the plane, $\Omega^{-2 / 3}$ was used as an average in the present experiment. The surface tensions in dry and moist hydrogen were estimated from the values measured by Hondros ${ }^{(10)}$ for $\delta$-iron at $1683 \mathrm{~K} ; 2.0 \mathrm{~J} \cdot \mathrm{m}^{-2}$ for dry hydrogen and $1.7 \mathrm{~J} \cdot \mathrm{m}^{-2}$ for moist hydrogen.

\section{Results}

\section{The surface self-diffusivity in dry hydrogen}

Photograph 1 shows the interferograms of grain boundary grooves developed at $1163 \mathrm{~K}$ for 5,20 and $100 \mathrm{~h}$, respectively. As shown in Fig. 2, the logarithmic plot of the groove width against annealing time resulted in a straight line, the slope of which was equal to 0.246 . As seen in eq. (1), the slope of $1 / 4$ indicates that the development of grain boundary groove has been controlled by surface diffusion. If the development of groove is controlled by volume diffusion or evaporation-condensation mechanism, the slope of the straight line should equal to $1 / 3$ or $1 / 2$ respectively, as shown by Mullins ${ }^{(9)}$.

The same annealing procedures were repeated at 1123 and $1073 \mathrm{~K}$, and the results obtained are summarized in Fig. 2.

\section{The surface self-diffusivity in moist hydrogen}

The first measurement in moist hydrogen was conducted at $1073 \mathrm{~K}$ and the results are shown in Photo. 2 and Fig. 3. As shown in Fig. 3, B, in the first run at $1073 \mathrm{~K}$ the logarithmic plot of the groove width and annealing time did not result in a straight line. Just before this run, the specimen had been used for the experiment in dry hydrogen at $1073 \mathrm{~K}$. The specimen, therefore, had been equilibrated with the oxygen potential of dry hydrogen $\left(P_{\mathrm{O}_{2}}=10^{-23.1} \mathrm{~Pa}\right.$ at $1073 \mathrm{~K})$. When this specimen was annealed in moist hydrogen $\left(P_{\mathrm{O}_{2}}=10^{-16.8} \mathrm{~Pa}\right.$ at $\left.1073 \mathrm{~K}\right)$, with higher oxygen potential, then the adsorption and dissolution of oxygen into iron should 

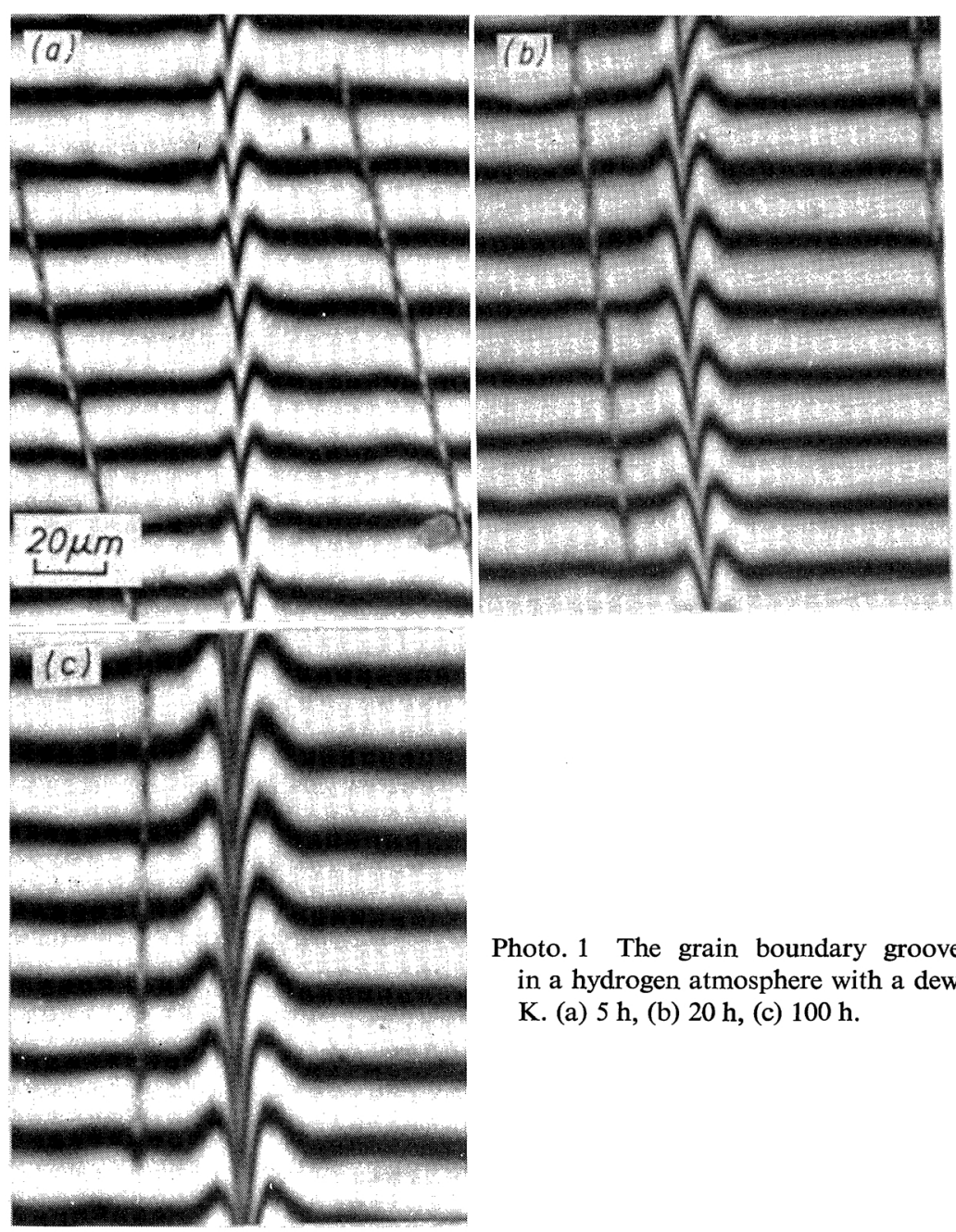

Photo. 1 The grain boundary grooves at $1163 \mathrm{~K}$ in a hydrogen atmosphere with a dew point of 213 K. (a) $5 \mathrm{~h}$, (b) $20 \mathrm{~h}$, (c) $100 \mathrm{~h}$.

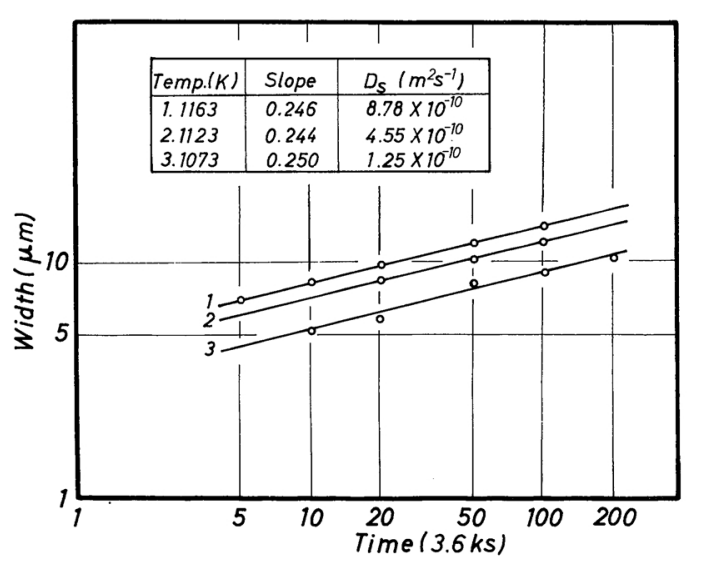

Fig. $2 \log w$ vs $\log t$ in a hydrogen atmosphere with a dew point of $213 \mathrm{~K}$. occur at first and this dissolution reaction on the surface would affect the rate of development of grain boundary groove.

To verify this inference, the specimen was deoxygenated again by annealing in dry hydrogen for $200 \mathrm{~h}$ and then diffusion-annealed under the same conditions (dew point $=286 \mathrm{~K}$, at $1073 \mathrm{~K}$ ). The logarithmic plot of the results, as shown in Fig. 3, C, deviated from a straight line in the same way as noted previously. These experiments clearly show that the deviation occurs when the specimen has not been previously equilibrated with atmosphere and therefore the adsorption and dissolution are taking place on the surface. Furthermore, it can be seen in Photo. 2 that the angle of the groove bottom increased with the annealing 


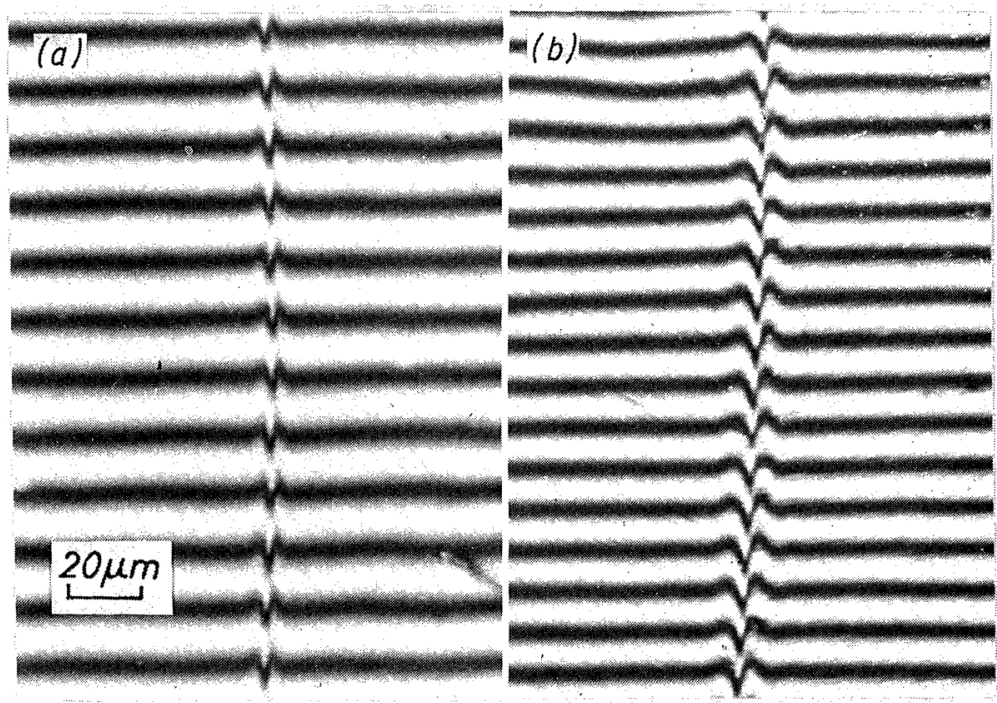

Photo. 2 The grain boundary grooves of the sample which was not equilibrated with hydrogen with a dew point of $286 \mathrm{~K}$ (at $1073 \mathrm{~K}$ ). (a) $5 \mathrm{~h}$, (b) $50 \mathrm{~h}$.

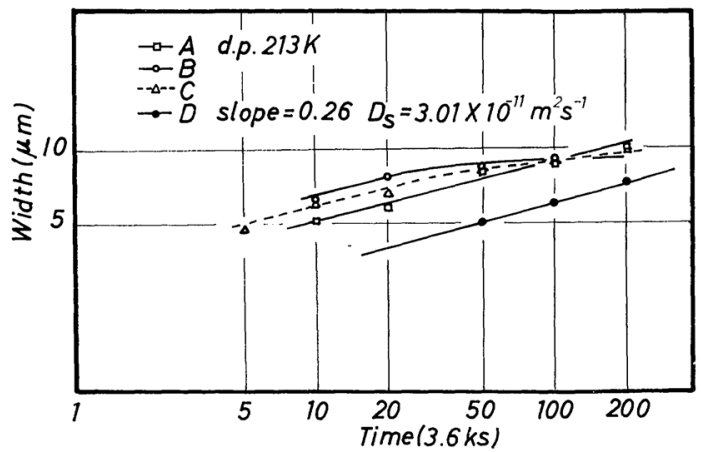

Fig. $3 \log w$ vs $\log t$ at $1073 \mathrm{~K}$ in a hydrogen atmosphere with a dew point of $286 \mathrm{~K}$.

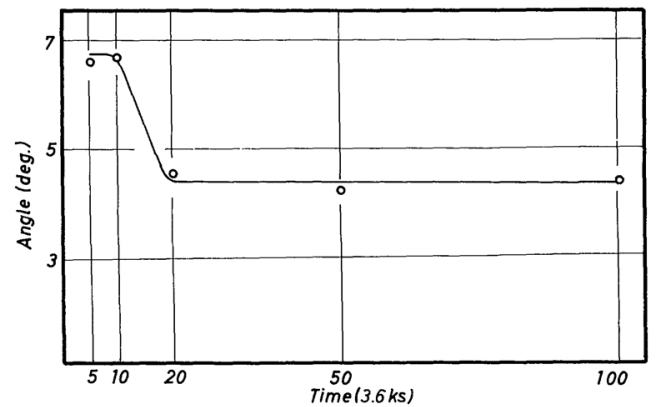

Fig. 4 The change of angles of grain boundary grooves. time. The angle of the grain boundary groove of the specimen was calculated from the angle on the interferogram by the following equation:

$$
\tan \beta=(2 l / \lambda M) \tan (\alpha / 2),
$$

where $\beta$ : angle between the tangent of the side plane at the groove bottom and the flat surface of the specimen

$l$ : distance between the interference lines on the photograph

$\lambda$ : wavelength of $\mathrm{Na}$-light

$M$ : distance between the marks of $100 \mu \mathrm{m}$ on the photograph

$\alpha$ : angle of the groove on the photograph.

The results are shown in Fig. 4. The angle of the grain boundary groove $\beta$ decreased sharply between 10 and $20 \mathrm{~h}$ annealing, where the growth rate of groove also begins to decrease. These results indicate that about $20 \mathrm{~h}$ has been required for the specimen to be equilibrated with moist hydrogen. The grain boundary tension is reduced by dissolution of oxygen and consequently the angle of the grain boundary groove $\beta$ should decrease until the new equilibrium state is established.

The fact that the growth rate of groove is large in these periods shows that the surface 

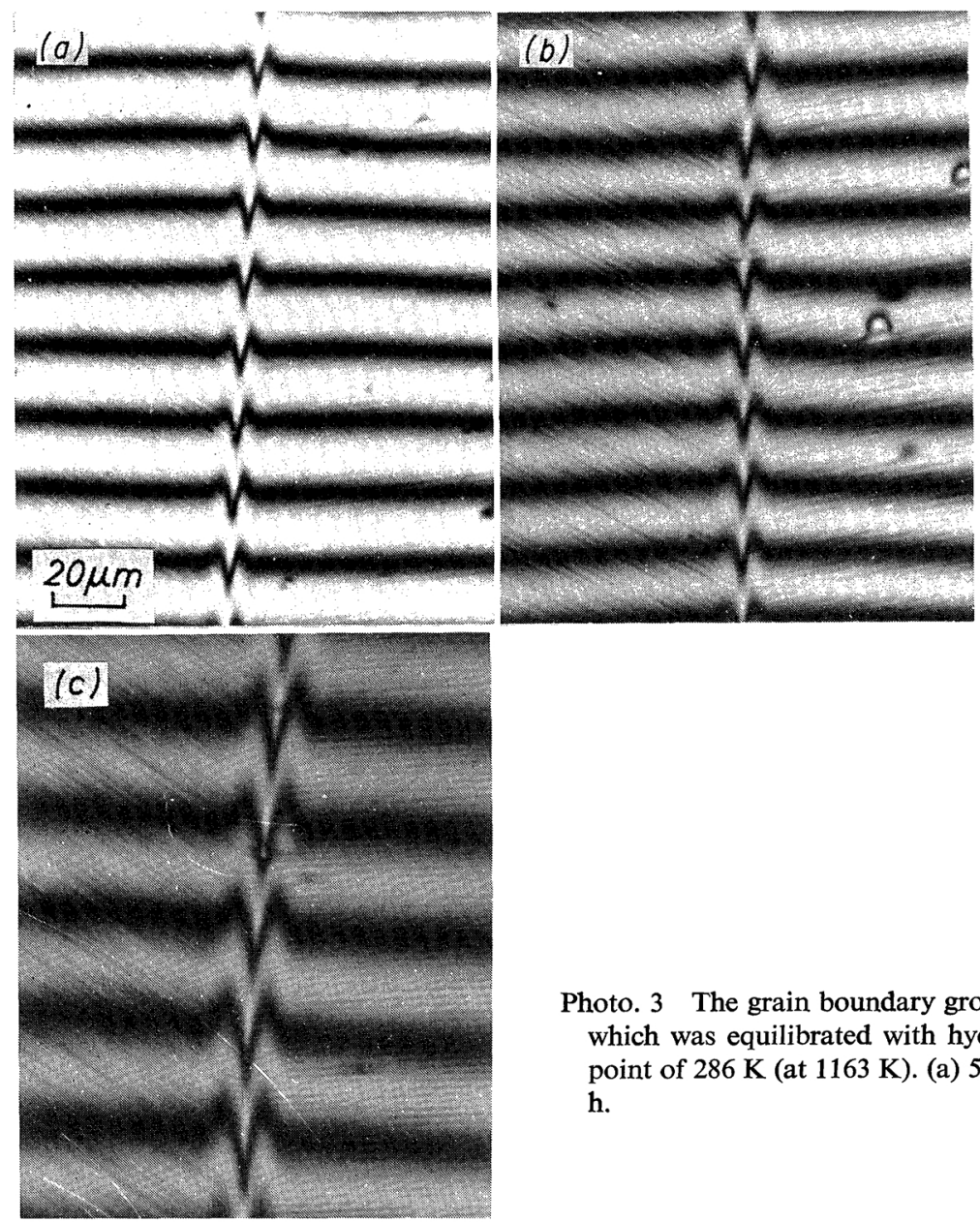

Photo. 3 The grain boundary grooves of the sample which was equilibrated with hydrogen with a dew point of $286 \mathrm{~K}$ (at $1163 \mathrm{~K}$ ). (a) $5 \mathrm{~h}$, (b) $20 \mathrm{~h}$, (c) 100 h.

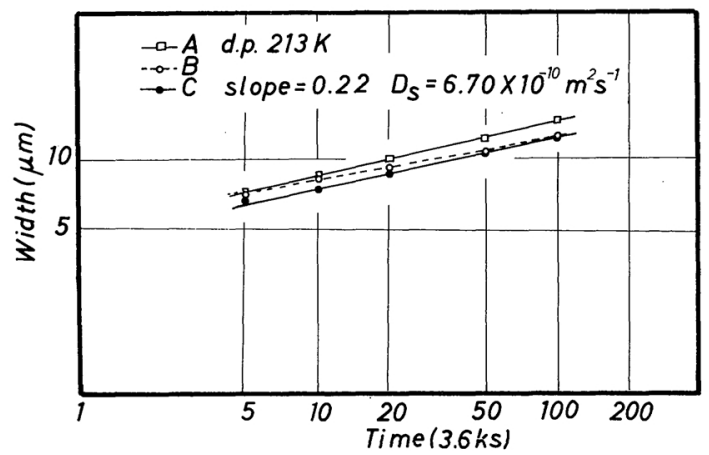

Fig. $5 \log w$ vs $\log t$ at $1163 \mathrm{~K}$ in a hydrogen atmosphere with a dew point of $286 \mathrm{~K}$.

self-diffusion coefficient $D_{s}$ is large on the surface where the dissolution of oxygen is in progress. In order to determine the surface self-diffusivity on the equilibrium surface, therefore, the specimen should be previously equilibrated with oxygen potential in the atmosphere. The results obtained with the specimen equilibrated with moist hydrogen are shown in Fig. 3, D. In this case, no dissolution of oxygen occurs, and the growth rate of groove and thus the surface self-diffusivity is much smaller than that in dry hydrogen.

The further experiments were carried out at $1163 \mathrm{~K}$ with the specimen which had been used in moist hydrogen at $1073 \mathrm{~K}$. The results are shown in Photo. 3 and Fig. 5. The results in the first run show that the groove widths in the short annealing time are larger than those in dry hydrogen but the grooving rate decreases with the annealing time. Although the loga- 


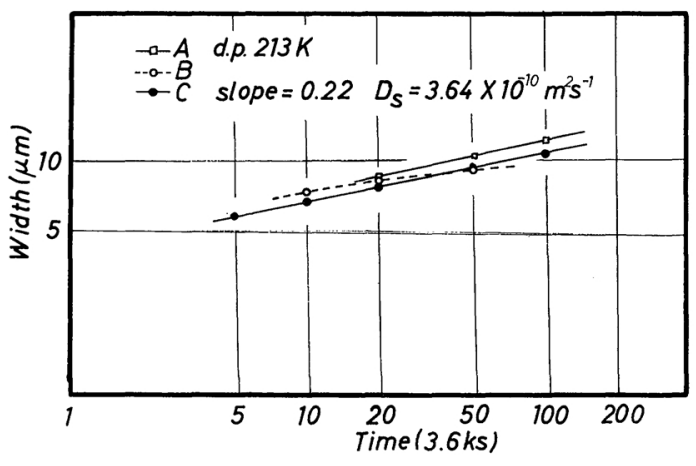

Fig. $6 \log w$ vs $\log t$ at $1123 \mathrm{~K}$ in a hydrogen atmosphere with a dew point of $286 \mathrm{~K}$.

rithmic plot of these groove widths results in a straight line, the slope of the line is much smaller than $1 / 4$, as shown in Fig. 5, B. The measurement was repeated under the same condition with the same specimen, then the straight line was observed as shown in Fig. 5, C. The groove widths in the second run were always smaller than those in dry hydrogen and the slope of the line was about $1 / 4$. In the first run, the specimen which had been equilibrated with an oxygen potential of $10^{-16.8} \mathrm{~Pa}$ at $1073 \mathrm{~K}$ was annealed in an oxygen potential of $10^{-14.9} \mathrm{~Pa}$ at $1163 \mathrm{~K}$, the saturated oxygen concentration would be different in both conditions. Therefore, the dissolution or release of oxygen on the surface would occur in the early stage of annealing. This situation would be the same as that of the first run in moist hydrogen at $1073 \mathrm{~K}$, and indeed the results obtained show the same tendency as those obtained at $1073 \mathrm{~K}$ (Fig. 3). The faceting was observed in moist hydrogen (Photo. 3).

Figure 6 shows the results obtained at $1123 \mathrm{~K}$. The first run, in which the specimen equilibrated with moist hydrogen at $1163 \mathrm{~K}$ was used, shows that the groove widths were larger in the initial stage and that the growth rate decreased later. In the second run where the equilibrium had been established between the specimen and the atmosphere, the groove widths were always smaller than those in dry hydrogen. In this case, the faceting was also observed.

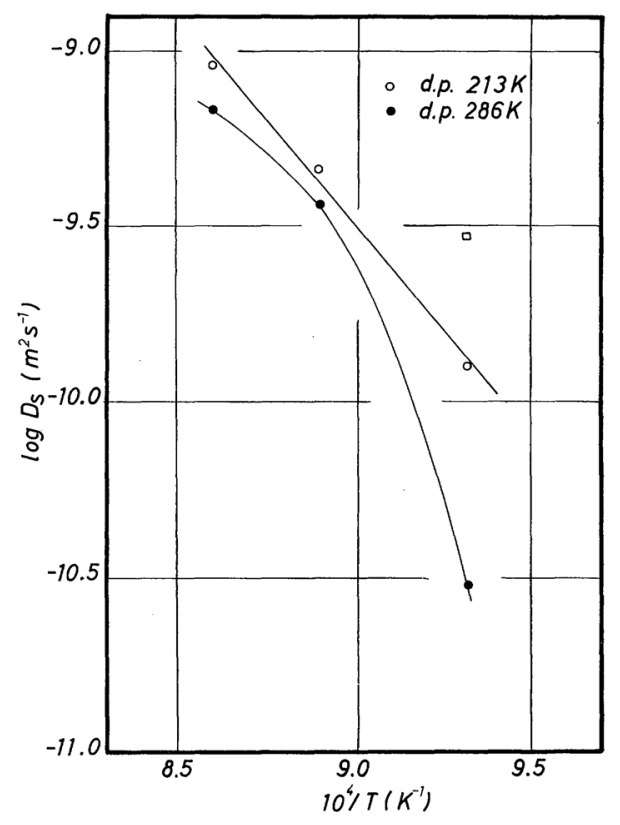

Fig. 7 Temperature dependence of diffusion coeffcients.

\section{Discussion}

The surface self-diffusivity obtained in the present experiment is summarized by Arrhenius plot in Fig. 7. The temperature dependence of the surface self-diffusivity in dry hydrogen can be expressed by

$$
D_{s}=10 \exp (-228 \mathrm{~kJ} / R T)\left(\mathrm{m}^{2} \cdot \mathrm{s}^{-1}\right) \text {. }
$$

The activation energy of $228 \mathrm{~kJ} / \mathrm{mol}$ agrees well with that obtained by Blakely et al. ${ }^{(1)}$ in vacuum $\left(1.3 \times 10^{-3} \mathrm{~Pa}\right)$, and is also close to the value estimated from the TLK (terrace, ledge, kink) model of the surface selfdiffusion $^{(12)}$. On the contrary, the temperature dependence of surface self-diffusivity in moist hydrogen is not represented by a straight line, the value decreases markedly at $1073 \mathrm{~K}$. The surface self-diffusivity on the surface where the oxygen dissolution was just in progress, was found to be extraordinarily large as depicted by $\square$ in Fig. 7. This value was calculated from the groove widths of 10 and $20 \mathrm{~h}$ annealing in the first run in moist hydrogen at $1073 \mathrm{~K}$, and was found to be ten times as large as the diffusivity obtained when the speci- 
men had been in equilibrium with the atmosphere.

The following mechanism is proposed in order to explain the present results.

In general, the surface self-diffusion can be interpreted as the migration of ad-atom or surface vacancy on the metal surface. The concentrations of these species are assumed to be determined by the equilibrium with kink site on the surface, and the migration is also assumed to take place along the chemical potential determined by curvature of the surface. Although the contribution of ad-atom to the surface self-diffusivity is claimed to be larger than that of surface vacancy at higher temperatures and smaller at lower temperatures $^{(12)}$, here we discuss the migration of ad-atoms as an example. The approximately same discussion can be extended to the migration of surface vacancies.

The flux of ad-atom $J_{a}$ along the surface can be given by the following equation ${ }^{(9)}$ :

$$
J_{a}=n_{a} v_{a}=-\left(m_{a} \gamma_{s} \Omega n_{a}\right)(\partial K / \partial s),
$$

where $m_{a}$ : mobility of ad-atom $\left(=D_{a} / k T\right)$

$n_{a}$ : number of ad-atom per unit surface area

$K$ : curvature of the surface

$s$ : distance along the surface.

By comparing this equation with that derived by Mullins, one can obtain

$$
D_{s}=\left(n_{a} / N\right) D_{a} \text {. }
$$

This means that the surface self-diffusivity is equal to the product of the mobility and density of ad-atoms. Accordingly, the constant $B$ in Mullins' equation (1) can be expressed as follows:

$$
B=D_{a} \gamma_{s} \Omega^{2} n_{a} / k T .
$$

As seen in the equation, the parameters which can be varied with atmosphere are the diffusion coefficient ot ad-atom $D_{a}$, the number of adatom per unit area $n_{a}$, and the surface tension $\gamma_{s}$.

In the present experiment, the surface selfdiffusivity in the equilibrium state decreased with an increase in adsorption of oxygen. It is not obvious, however, which parameter is dominantly responsible for the decrease in diffusivity. The change in $\gamma_{s}$ was at least taken into consideration in use of the value of surface tension by Hondros, though for more exact correction the measurement is required under the same conditions as diffusion measurement. The decrease, therefore, would be originated by the decrease in either mobility or surface density of ad-atom, or both. Since the adatoms are assumed to be in equilibrium with kink sites on the surface, the density of ad-atom would decrease if the kink sites are stabilized by adsorption of oxygen at the kink site. Furthermore, if the adsorbed oxygen forms a pair with ad-atom, then the mobility of ad-atom will also be markedly reduced. These two processes can reduce the surface selfdiffusivity and therefore can explain the present results, but it is not clear which is predominant in reducing surface self-diffusivity.

On the other hand, the rapid growth of the groove was observed in the early stage of annealing in the first run after the annealing temperature was changed $1073 \mathrm{~K} \rightarrow 1163 \mathrm{~K} \rightarrow$ $1123 \mathrm{~K}$ in the same atmosphere. This indicates that the surface self-diffusivity is remarkably enhanced on the surface where the dissolution or release of oxygen is taking place. Now let us consider the change in $n_{a}$ and $D_{a}$ under these situations. The rate of dissolution of oxygen into metals is generally assumed to be determined by the diffusion in metals and not by the surface reactions. Therefore, the number of adsorbed oxygen on the surface would be the same both in equilibrium state and in non-equilibrium state. Then the immobile pair of ad-atom and adsorbed oxygen would be formed to the same extent and thus the decrease in mobility of ad-atom would be expected to occur to the same extent. In the non-equilibrium state, however, the reactions such as dissociation-dissolution or association-release of oxygen are taking place on the surface, and accordingly the configuration of surface metal atoms would be much more disturbed than that in the equilibrium state, that is, the number of ad-atom $n_{a}$ would increase more than that in the equilibrium state.

If the increase in number of ad-atom is large enough to cancel the decrease in mobility of ad-atom, the surface self-diffusivity can increase more than that in the equilibrium state. 
The inference that the disturbance of surface atoms would increase by surface reactions can be approved by the experiments other than surface diffusion. White ${ }^{(13)}$ has measured the temperature dependence of surface tension of molten zinc and found that its surface entropy is larger when evaporation of zinc is taking place than when the equilibrium is established between vapor and melt. The surface entropy is an excess entropy of surface atoms over that of interior atoms, and thus the large surface entropy on the evaporating surface means that the disturbance of configuration and vibration of surface atoms are more intense than those on an equilibrium surface ${ }^{(14)}$. The negative surface entropy has been also reported for the surface of $\mathrm{Fe}-3 \% \mathrm{Si}$ alloy in high oxygen potential ${ }^{(15)}$. This results indicate that the disturbance of surface atoms would decrease by the adsorption of oxygen, and therefore that the concentration of ad-atoms or surface vacancies can be assumed to be lowered by the oxygen adsorption. This corresponds to the present experimental results that the surface self-diffusivity decreases on the surface where the equilibrium adsorption of oxygen is established.

The decrease in surface self-diffusivity in moist hydrogen is very remarkable at $1073 \mathrm{~K}$. The marked decrease at lower temperatures has also been observed for the surface selfdiffusivity of gold $^{(16)}$, which was interpreted as the pinning of ledge by the impurities adsorbed at the ledge site. The similar mechanism for ledge movement has been applied to the formation of facet or dissolution of solid into solution ${ }^{(17)}$. In the present experiment, faceting was observed in moist hydrogen as shown in Photo. 3, but did occur not only at $1073 \mathrm{~K}$ but also at higher temperatures. Therefore, the ledge pinning mechanism is not sufficient for the marked decrease in surface self-diffusivity at lower temperatures. Gjostein $^{(16)}$ has also reported that the straight line in the Arrhenius plot extends down to the lower temperature if the specimen is once pre-annealed at high temperatures to achieve the more complete desorption of impurities from surface. Consequently, it would be certain that the marked decrease in diffusivity at lower temperatures should be caused by adsorption of impurities on the surface, though the detailed mechanism is not obvious. The marked decrease at $1073 \mathrm{~K}$ in the present experiment could also be attributed to the same mechanism. The other mechanism for the decrease in diffusivity has been proposed ${ }^{(18)}$, but no general conclusion has been drawn as yet.

As mentioned previously, the effect of adsorption of oxygen, sulfur or halogens on the surface self-diffusivity has been investigated for silver, copper or iron. In these experiments, however, it is not certain whether or not the specimen has been equilibrated with the atmosphere. Further investigation will be necessary for evaluating the effect of adsorption.

\section{Summary}

The effect of oxygen potential on the surface self-diffusivity of $\alpha$-iron was investigated by grain boundary grooving method. The results obtained here are summarized as follows.

(1) In dry hydrogen (dew point $=213 \mathrm{~K}$ ), the surface self-diffusivity can be expressed by the following equation:

$$
\begin{gathered}
D_{s}=10 \exp (-228 \mathrm{~kJ} / R T)\left(\mathrm{m}^{2} \mathrm{~s}^{-1}\right) \\
(1073 \mathrm{~K}-1163 \mathrm{~K}) .
\end{gathered}
$$

(2) In moist hydrogen (dew point $=286 \mathrm{~K}$ ), the surface self-diffusivity is varied, depending on whether or not the specimen has been equilibrated with the atmosphere. When the specimen has been equilibrated with moist hydrogen, the surface self-diffusivity is smaller than that in dry hydrogen, and the decrease is remarkable at $1073 \mathrm{~K}$. When the specimen has not been equilibrated, the dissolution or release of oxygen occurs on the surface, and on this surface the very large surface diffusivity is observed.

These results can be explained by the stabilization of kink sites and the formation of immobile ad-atom by adsorption of oxygen. The marked decrease in surface self-diffusivity in moist hydrogen at $1073 \mathrm{~K}$ could be attributed to the adsorption of impurities.

\section{REFERENCES}

(1) K. Nii: Z. Metallk., 61 (1970), 935.

(2) G. E. Rhead: Acta Met., 13 (1965), 223. 
(3) J. Perdereau and G. E. Rhead: Surface Sci., 7 (1967), 175.

(4) F. J. Bradshaw, R. H. Brandon and C. Wheeler: Acta Met., 12 (1964), 1057.

(5) H. E. Collins and P. G. Shewmon: Trans. Met. Soc. AIME, 236 (1966), 1354.

(6) J. Henrion et G. E. Rhead: C. R. Acad. Sc. Paris Série B, 267 (1968), 958.

(7) F. Delamare and G. E. Rhead: Surface Sci., 28 (1971), 267.

(8) R. Zahn: Doctoral Thesis, Bergakademie Clausthal, (1964).

(9) W. W. Mullins: J. Appl. Phys., 28 (1957), 333.

(10) E. D. Hondros: Acta Met., 16 (1968), 1377.

(11) J. M. Blakely and H. Mykura: Acta Met., 11 (1963), 958.
(12) N. A. Gjostein: Surfaces and Interfaces, 1, Ed. by J. J. Burk, N. L. Reed and V. Weiss, Syracuse Univ. Press, Syracuse, N.Y., (1967), p. 271.

(13) D. W. G. White: Trans. Met. Soc. AIME, 236 (1966), 796.

(14) R. H. Ewing and B. Chalmers: Surface Sci., 31 (1972), 161.

(15) E. D. Hondros and L. E. H. Stuart: Phil. Mag., 17 (1968), 711.

(16) N. A. Gjostein: Adsorption et croissance cristalline, Colloques Intern, CNRS, Nancy, (1965), p. 97.

(17) A. J. Moore: Metal Surfaces: Structure, Energetics and Kinetics, ASM, (1963), p. 155.

(18) M. McLean and J. P. Hirth: Surface Sci., 12 (1968), 177. 